Portland State University

PDXScholar

Mechanical and Materials Engineering Faculty

Publications and Presentations

$7-1-2010$

\title{
Developing Sustainable Life Support System Concepts
}

\author{
Evan A. Thomas \\ Portland State University
}

Follow this and additional works at: https://pdxscholar.library.pdx.edu/mengin_fac

Part of the Systems Engineering Commons

Let us know how access to this document benefits you.

\section{Citation Details}

Thomas, E., Developing Sustainable Life Support System Concepts, 40th International Conference on Environmental Systems, Barcelona, Spain, 2010.

This Article is brought to you for free and open access. It has been accepted for inclusion in Mechanical and Materials Engineering Faculty Publications and Presentations by an authorized administrator of PDXScholar. Please contact us if we can make this document more accessible: pdxscholar@pdx.edu. 


\title{
Developing Sustainable Life Support System Concepts
}

\author{
Evan A. Thomas, Ph.D. ${ }^{1}$ \\ NASA-Johnson Space Center, Houston, Texas 77058
}

\begin{abstract}
Sustainable spacecraft life support concepts may allow the development of more reliable technologies for long-duration space missions. Currently, life support technologies at different levels of development are not well evaluated against each other, and evaluation methods do not account for long-term reliability and sustainability of the hardware. This paper presents point-of-departure Sustainable System Mass (SSM) evaluation criteria for life support systems that may allow more robust technology development, testing, and comparison. An example sustainable water recovery system concept is presented.
\end{abstract}

\section{Introduction}

Q ustainability is the capacity to endure. Long-duration space flight, as anticipated for moon, Mars and other $\checkmark$ planetary missions, will require hardware that is less prone to failure and generally more rigorous and sustainable than the current state of the art. Sustainable Environmental Control and Life Support Systems (SECLSSs) may be developed to function for long periods of time in harsh environments, with limited maintenance and resupply. Water recovery, air revitalization, habitation, food, and power systems may benefit from considering sustainability a design goal.

The SECLSS project is designed to develop rough life support system architectures, evolve technology concepts, and collaborate with NASA partners to consider long-term sustainability as a design driver for life support systems. One example of an application requiring innovation is wastewater management, wherein wastewater fouling is accommodated by the design of fluid management hardware. This paper provides an overview of the SECLSS project concept and proposed sustainability evaluation criteria for Environmental Control and Life Support System technologies, and details a preliminary example technology for water recovery on a planetary outpost.

\section{Background}

President Obama has directed NASA and its partners to develop sustainable life support systems. On April 15, 2010, the President's speech at NASA Kennedy Space Center, 'Remarks by the President on Space Exploration in the $21^{\text {st }}$ Century', included these statements: "And we will extend the life of the International Space Station likely by more than five years, while actually using it for its intended purpose: conducting advanced research that can help improve the daily lives of people here on Earth, as well as testing and improving upon our capabilities in space. This includes technologies like more efficient life support systems that will help reduce the cost of future missions," and, "Our goal is the capacity for people to work and learn and operate and live safely beyond the Earth for extended periods of time, ultimately in ways that are more sustainable and even indefinite."

This vision is directly in-line with the concepts pursued in this paper. For example, a long-term planetary outpost will require sustainable life support technologies that are capable of functioning for years with minimum resupply and maintenance. While life support resources such as water and air will remain in short supply, the availability of gravity, energy, and natural resources on the planetary surface will allow for innovation in the design of outpost technologies, potentially including the adoption of terrestrial technologies previously not feasible for short-duration microgravity flight.

As missions become extended in duration and move toward more self-reliant operations, new demands are placed on life support system designs. Thus far, all indications have suggested that planetary outpost water recovery systems will be evolved from current spacecraft technologies, including urine pretreatment, distillation, and brine dewatering ${ }^{1}$. However, these technologies were developed for microgravity compatibility, and may carry undesirable fouling and failure mode heritage from this environment. For example, it is well recognized that water handling systems used in spacecraft are prone to failure caused by biofouling and mineral scaling, which can clog mechanical systems and degrade the performance of capillary-based technologies. Recent challenges with the urine processing assembly on the International Space Station point to urine precipitate fouling of the mechanical hardware.

\footnotetext{
${ }^{1}$ Project Manager, Life Support and Habitability Systems Branch, Johnson Space Center, 2101 NASA Parkway,
} Houston, Texas, 77058, Mail Code EC3, AIAA Member Grade MB. 


\section{Sustainable Environmental Control and Life Support System Concepts}

The concepts packaged within the Sustainable Environmental Control and Life Support System (ECLSS) project are not new. Spacecraft engineers have always been concerned with reliability, maintainability, robustness, and performance. However, competing requirements and mechanisms of technology development have sometimes resulted in programs focusing on existing, complex technologies that have evolved for specific reasons and constraints. These technologies may, in fact, not be particularly sustainable or appropriate for longer-duration space missions. Instead, the original constraints and requirements can be revaluated based on actual expected mission conditions, and technology development can be integrated with other architecture considerations appropriate for the expected mission profile. For example, a life support technology developed for the International Space Station's microgravity environment may or may not be appropriate for a similar use on a planetary outpost, particularly when the hardware is necessarily complex to manage fluids in microgravity. A partial-gravity environment can dramatically simplify the technology, and likely improve sustainability.

ECLSS subsystems are often evaluated using equivalent system mass (ESM), which sums the real mass of a system with mass penalties for volume, power, cooling, crew time, and logistics. ESM was developed specifically for spacecraft ECLSS subsystems. However, ESM does not currently directly account for reliability and other sustainability considerations, but instead assumes a similar level of potential failures between technologies.

The developers of ESM state, "Ideally, an effort should be made to adjust the design of subsystems or systems in order to bring them to similar levels of potential failures ... an effort should be made to design subsystems to similar levels of reliability by taking into account the mean time before failure. In reality, a lack of data may prevent ESM from accounting for reliability and safety specifications ... If this is the case, the researcher's expertise on the matter may then be used to make reasonable adjustments in ESM in order to compare technologies."

Therefore, ESM inherently relies on technology developers to provide candid input on reliability, and requires operational experience to evaluate failures. ESM has no mechanism to compare technologies at different Technology Readiness Levels (TRL) and, as such, currently has limited capability to allow the evaluation of a range of potential technologies. ESM also assumes the technologies meet identical reliability and technical requirements, "Comparison of systems or subsystems with ESM is only suitable where the systems or subsystems satisfy identical requirements, including levels of safety and reliability." ${ }^{2}$

Other technology and architecture developers have struggled with these same limitations in evaluating the state of the art alongside promising technologies, "ESM is the sum of real masses and mass penalties for cost factors judged to be significant for life support: volume, power, cooling, manpower, and logistics. ... Thus it does not consider flight readiness (TRL) [or] requirements options. It is taken as a given that capacity and safety requirements are met, and that whatever is necessary to keep the system running for the duration of the mission ([e.g.,] spares, makeup gas, crew time) is included in ESM.",

These assumptions of TRL and reliability are at the very least limiting for ESM evaluations. This paper proposes a point of departure for adding sustainability criteria to ESM calculations that may allow comparison of technologies at different TRLs, and may identify commonalities and best practices to enable the development of more sustainable ECLSS technologies.

The United States Green Building Council Leadership in Energy and Environmental Design (LEED) Green Building Rating System is used as an inspiration for these criteria. LEED was designed to standardize environmental sustainability evaluation criteria for new and remodeled buildings. The LEED concept uses a point-based system to award a score to a design based on several categories, including site selection, water use, energy use, materials selection, indoor air quality, use of innovative technologies, and consideration of local conditions. The criteria listed below, which employ the spirit of this evaluation tool as a guide, are proposed for integration with ESM calculations. The author fully recognizes these are only points of departure, and the tabulated points presented are for discussion purposes only. The intent is to encourage a discussion of sustainable practices for ECLSS technologies.

\section{A. Simplest Feasible Design}

Striving for simplicity in design is a long-standing engineering goal. Spacecraft system engineers are trained no differently; however, programmatic considerations often drive technology to more complex and, therefore, often less sustainable configurations. Unfortunately, often there is no strong push-back against this progression. It is suggested here that evaluation criteria can be standardized to evaluate the compliance of a technology with the most simplified design that complies with appropriate requirements. An initial representation of these criteria is presented below.

a) Appropriate Requirements - The process of developing hardware requirements is necessarily inclusive of many stakeholders, who each have a niche consideration. However, because of this, project requirements can 
become unwieldy, requiring technology to grow in complexity to respond to all requirements. Therefore, it is suggested that program managers work with project managers to regain control over requirements creep and develop the process authority to reject unnecessary requirement burdens. Technologies that successfully simplify their requirements will likely be more sustainable.

b) Complexity - The technical complexity of a subsystem is often driven by design requirements. However, complexity also often results in an increasing likelihood of debilitating failures. By identifying hardware complexity separately from performance, a more comprehensive understanding of mission capability may be developed.

c) Reliance on Controlled Operating Conditions - Performance and operational requirements drive subsystem design. Engineers appreciate well-defined environmental conditions that allow the development of technologies with high confidence that they will meet defined requirements. However, the more narrowly defined the requirements, the more likely the operational environment will exceed these requirements. Therefore, it is appropriate to evaluate how reliant a design is on narrowly defined requirements, and whether the system can accommodate a broader range of operational conditions. For example, some wastewater management technologies rely on near-perfect operation of oxidizing pretreatment chemicals. Should the pretreatment fail to prevent wastewater fouling, some technologies will degrade significantly and may fail. Other designs may allow for accommodation of such conditions, however.

d) Dissimilar Redundancy, and Degraded Performance - When expected and unexpected failure modes manifest during flight, redundancies are expected to allow the safe execution of a mission. The capacity for technology manifest of unexpected failures suggests that dissimilar redundancies, including technologies that may offer a lower level of performance, may be a valuable approach. Similarly, technologies that can offer degraded but nonzero performance may prevent emergencies.

e) Integration - Comprehensive and thoughtful consideration by architecture and technology developers upstream of hardware builds can prevent integration issues, in which one technology is wholly dependent on another's performance. Likewise, comprehensive understanding of the range of inputs to be expected by, and outputs expected of, the technology may result in more robust performance.

f) Uptime $\times$ Key Performance Parameter (KPP) - Designs are often driven to higher performance standards. However, higher performance may also yield more complex and failure-prone designs. Therefore it is important to consider both performance and uptime. The factor of uptime percent to the functional KPP percent may be an appropriate tool to compare technologies. For example, is a water recovery system with $90 \%$ recovery that has $60 \%$ uptime better or worse for a mission than a system with $80 \%$ recovery but a $90 \%$ uptime $(0.54$ factor vs. 0.72)?

g) TRL $\times$ Critical Failure Frequency $(\mathbf{C F F})-$ TRL is used as an approximation of the maturity of a given design. The general assumption is made that higher-TRL-level systems are more reliable and "validated." However, as illustrated by other parameters and examples listed here, higher-TRL-level technologies do not necessarily result in more robust or appropriate technologies. Caution should thus be taken to appropriately consider TRL when comparing technologies against one another. One potential way of evaluating the true value of a higher TRL technology is to multiply the TRL by the frequency of debilitating failures. This will better allow the sustainability of technologies at different TRLs to be compared. For example, if a TRL 9 technology that is ostensibly flight-proven has a failure taking the system out of service twice a year, the TRL $\times$ CFF factor is 18 . In contrast, a laboratory-based proof-of-concept TRL 6 technology that has a failure every 3 months of components under test would have a TRL $\times$ CFF factor of 24 . Should this system have failures only twice a year, the TRL $\times$ CFF would be 12 . Therefore, the lower the TRL $\times$ CFF factor, the higher the likelihood of a sustainable, robust design. Of course, a lower TRL hardware is not normally tested in a representative environment. Therefore, this TRL x CFF parameter will only be effective when environmental testing is matched, as described in the following section.

\section{B. High-fidelity Environmental Testing}

In addition to technical design considerations, it is possible that more sustainable spacecraft life support systems might also be developed by reconsidering the methods in which they are typically tested, normally under highly controlled conditions. The goal is generally to conduct defined tests that produce predictable and reproducible results akin to how basic scientific research is carried out. Once these systems are in space, however, they often fail in complex, unforeseen ways leaving engineers frustrated and the systems in disuse.

Designing, testing, and evaluating spacecraft life support systems is more an engineering challenge than a basic science research challenge. Engineers are generally more interested in how well a given system meets operational requirements rather than in examining fundamental processes. Testing protocols, therefore, should be adjusted to 
reflect this goal. Systems should not need to be fully characterized under precise and controlled environments; rather results from complex and compounded conditions within defined boundaries should be compared to stated performance requirements. Suggested basic elements of this approach are as follows:

1) Set performance requirements and evaluation criteria for desired technology

2) Define reasonable envelope of expected operational environmental conditions

3) Evaluate multiple technologies within this expected operational envelope

4) Escalate, expand, and compound the envelope as technologies mature

5) Evaluate results against performance requirements and technology capabilities

While this approach may seem like standard engineering practice, it is in fact a departure from the methods in which most spacecraft life support systems are tested, perhaps a consequence of the rarity and expense of "in-space" field testing. Specifically, today's typical approach is to control the testing environment in such a way that any particular requirement is evaluated in relative isolation. For example, ground tests with fluid systems often use ersatz, over-simplified conditions that do not fully represent the actual environment that produces appropriate complex surface conditions in which fouling occurs. Engineering performance tests consequently should be less concerned with fully characterizing a single parameter in favor of gaining confidence in the system's overall robustness and sustainability across a range of expected conditions.

This approach will result in more sustainable spacecraft systems. Through designing, testing, and evaluating technologies based on the truly complex environments to which they will be exposed, designers can introduce methods that will enable technologies to be more robust, easily maintained, and recoverable. For example, a system need not be designed for the worst-case scenario in each direction of the environmental envelope, as long as the system can recover from degraded performance. Should a spike in a particular condition cause a failure or a degraded performance, that system should be able to both a) identify that an alarm condition has occurred, and b) allow for recovery to operational performance with minimal consumable or crew cost. Additionally, by allowing engineers to design for end-of-life performance, systems will have more predicable operational and maintenance characteristics. Several evaluation criteria for the testing environment are proposed below:

a) Appropriateness for Environment - Subsystems are initially driven by flight requirements. However, the long development profile of life support hardware can result in technologies that ultimately are not fully appropriate for their operational conditions. For example, adapting microgravity-compatible technologies for a planetary surface may not be the most technologically appropriate solution, resulting in needlessly complex and failure-prone hardware being used in an environment in which simplified hardware could be used instead. An evaluation of the technology candidate for appropriateness in the operational environment may identify potential alternatives, improvements, or simplifications.

b) Failure Types and History - Development units, ground testing, and flight operations identify component and system-level issues that can range from nuisances to maintenance concerns to debilitating failures. The nature, impact, and understanding of these failures can provide an important dimension to technology evaluation. For example, if a valve fails on a breadboard system in a laboratory, this may be an expected and well-characterized failure. However, if a flight hardware systems fails when unexpected kinds of wastewater fouling have clogged a fluid management system and this failure was not expected or well characterized, the fundamental design may be reevaluated.

c) Testing History and Rigor - Engineering performance tests consequently should be less concerned with fully characterizing a single parameter in favor of gaining confidence in the overall robustness and sustainability of a system across a range of expected conditions.

d) Consumables - Normally, ELCSS consumables (e.g., make-up gases, balance water, packaging, on on-orbit replaceable units (ORUs)) are accounted for by designers and planners with some margin. This planning does not account for off-nominal performance or unexpected failures, however. Because of this, the ESM calculation for consumables does not accurately reflect the dramatic increase in cost associated with crew time and emergency resupply. A more comprehensive understanding of technology robustness may yield a more accurate estimation of consumables.

\section{Sustainable System Mass (SSM)}

These point-of-departure criteria are tabulated in Table 1 with Sustainable System Mass (SSM) point placeholders presented for the reader to consider the value of each parameter. The author has not attempted to assign values to these parameters for several reasons. First, collecting relevant historic data and deducing trends to develop appropriate scoring will be a collaborative process with various stakeholders and hardware developers. At this point, assigning values will distract from the intent to elicit conversation on the concept of sustainable life support 
hardware. Secondly, it is well recognized that any scoring system, including ESM and LEED, can lead to counterproductive results. For example, at NASA's White Sands Test Facility, riding bicycles on-site is against the rules for safety considerations. Yet, bike racks have been added at some buildings to gain points on the LEEDs rating system. Likewise, rainwater catchment systems on Las Vegas casinos hardly lead to long term sustainability.

Therefore, the author hopes that these guidelines and scorecard will simply elicit conversation and enable the development of appropriate design and evaluation criteria that push technologies toward improved sustainability. It is suggested that a revised table tailored appropriately for each technology with general scorecard values assigned to SSM parameters could then be integrated with ESM calculations, with each SSM point representing one kg of ESM. Table 1 enables the reader to consider the relative value of each parameter listed.

Table 1. Potential Sustainable System Mass (SSM) Parameters for Equivalent System Mass (ESM)

\begin{tabular}{|c|c|c|}
\hline Parameter & Evaluation Criteria & $\begin{array}{l}\text { Sustainable System Mass } \\
\text { (SSM, equivalent to ESM) }\end{array}$ \\
\hline \multicolumn{3}{|l|}{ Simplest Feasible Design } \\
\hline $\begin{array}{ll}\text { a. } & \text { Appropriate } \\
& \text { Requirements }\end{array}$ & $\begin{array}{l}\text { Evaluation by technology developers that } \\
\text { requirements are simplified }\end{array}$ & ${ }^{(+)} \_{ }^{(-)}{ }_{-}$N/A \\
\hline \multirow{2}{*}{ b. Complexity } & Dynamic components & $(+) \ldots(-) \ldots N / A$ \\
\hline & Static control of fluids & $(+) \ldots(-) \ldots N / A$ \\
\hline $\begin{array}{ll}\text { c. } & \text { Reliance on } \\
& \text { Controlled } \\
& \text { Operating } \\
\text { Conditions }\end{array}$ & $\begin{array}{l}\text { Independent evaluation of input/output } \\
\text { assumptions from integrated hardware }\end{array}$ & $\begin{array}{l}(+) \ldots(-) \ldots N / A \\
\text { per parameter defined } \\
\text { within _\% }\end{array}$ \\
\hline \multirow{2}{*}{$\begin{array}{ll}\text { d. } & \text { Dissimilar } \\
& \text { Redundancy and } \\
\text { Degraded } \\
\text { Performance } \\
\end{array}$} & Dissimilar redundancy capability & $(+) \ldots(-) \ldots N / A$ \\
\hline & $\begin{array}{l}\text { Capability to operate at degraded performance } \\
\text { standard under simplified operating conditions }\end{array}$ & ${ }^{(+)} \_(-) \_N / A$ \\
\hline e. Integration & Number of systems to be integrated & ${ }^{(+)}{\underset{\text { per system }}{(-)}}^{\mathrm{N} / \mathrm{A}}$ \\
\hline f. Uptime $\times$ KPP & Use of KPP typical for similar systems & $\begin{array}{l}(+) \\
\text { Factor } x_{\text {_ }}^{(-)} \text {points }\end{array}$ \\
\hline g. TRL $\times$ CFF & Frequency of critical failures & $(+) \ldots(-) \ldots N / A$ \\
\hline \multicolumn{3}{|c|}{ High-fidelity Environmental Testing } \\
\hline \multirow{2}{*}{$\begin{array}{l}\text { a. Appropriateness } \\
\text { for Environment }\end{array}$} & $\begin{array}{l}\text { Increased complexity of design for microgravity } \\
\text { compatibility when intended for planetary surface }\end{array}$ & ${ }^{(+)}{ }^{(-)}{ }^{(-)}{ }^{\mathrm{N} / \mathrm{A}}$ \\
\hline & Consideration of all inputs and outputs to system & $(+) \ldots(-) \ldots N / A$ \\
\hline \multirow{6}{*}{$\begin{array}{l}\text { b. Failure Types and } \\
\text { History }\end{array}$} & Component Level & $(+) \ldots(-) \ldots N / A$ \\
\hline & System Level & $(+) \ldots(-) \ldots N / A$ \\
\hline & Fouling & $(+) \ldots(-) \ldots N / A$ \\
\hline & Anticipated Failures & $(+) \quad(-) \ldots N / A$ \\
\hline & Unexpected Failures & $(+) \ldots(-) \ldots N / A$ \\
\hline & Restoration without special tooling or resupply & $(+) \ldots(-) \ldots N / A$ \\
\hline \multirow{3}{*}{$\begin{array}{l}\text { c. Testing History } \\
\text { and Rigor }\end{array}$} & Appropriately defined environmental testing & $(+) \ldots(-) \ldots N / A$ \\
\hline & Independent validation testing & $(+) \ldots(-) \ldots N / A$ \\
\hline & Compliance with sustainable testing criteria & $(+) \ldots(-) \ldots N / A$ \\
\hline \multirow{4}{*}{ d. Consumables } & Anticipated consumables & $\begin{array}{l}(+) \\
\text { per component }\end{array}$ \\
\hline & Unexpected replacement needs & $\begin{array}{c}(+) \\
\text { per component }\end{array}$ \\
\hline & Maintenance requirements & $\begin{array}{l}(+) \\
\# \text { hours per year }\end{array}$ \\
\hline & In-situ resource utilization (ISRU) & $(+) \ldots(-) \ldots N / A$ \\
\hline
\end{tabular}

American Institute of Aeronautics and Astronautics 


\section{Example Sustainable Water Recovery System Concept}

An example ECLSS technology category that may benefit from increased attention on sustainability are systems that manage water and wastewater. Development of spacecraft life support hardware over the past few decades has focused primarily on microgravity applications, with sophisticated designs usually constrained by volume, mass, and power limitations. In particular for two-phase gas/liquid separation in microgravity, centripetal acceleration or capillary action is used to remove liquids without the aid of gravity-driven buoyancy. These systems have often been prone to failure due to fluid fouling caused by biological reactions or mineral scaling, which can clog mechanical systems and degrade the performance of capillary-based systems. In turn, these failures can cause increased maintenance cost and overall crew labor burden. Current spacecraft Water Recovery Systems (WRS) rely on oxidizing pretreatment chemicals to stabilize wastewater, to prevent fouling.

Instead of adapting this approach, a planetary outpost will have other, potentially beneficial characteristics that can be considered during system design. For example, unlike orbiting spacecraft, a lunar outpost will exist in a fractional Earth gravity environment $\left(\sim 0.166 g_{o}\right)$ with abundant natural resources including lunar regolith, vast open surfaces, and plentiful sunlight. Gravity can, at worst, make complex microgravity-compatible technologies unnecessary and, at best, be advantageously used in a wastewater recovery process. Meanwhile, the outpost may not have ready access to Earth resupply, making consumables and maintenance of greater concern when conducting design trade studies. Lunar surface conditions are perhaps more analogous to the terrestrial environment than to microgravity space flight. For these reasons, the appropriate technology development approach for lunar outpost hardware is likely to be adapting terrestrial technologies for use in a hypo-gravity environment rather than modifying microgravity space flight technologies.

A planetary outpost may therefore consider using simple and robust terrestrial technologies such as media filters, solar disinfection, and distillation to recover water, taking advantage of the ready availability of planetary regolith, gravity, and solar energy. Rather than stabilizing wastewater such as urine for disposal, the wastewater could thus be encouraged to foul the media and form biofilms and precipitates that can then be filtered and the water reclaimed for future use. This concept is detailed in a 2009 paper from this conference. ${ }^{4}$

\section{Conclusion}

Long-duration space missions, such as expected for a planetary outpost or a Mars transit, will drive the need for hardware that is less prone to failure and generally more robust and sustainable. Technology developers currently are constrained by complex requirements, operational environments, and development momentum. This can lead to technologies that are more complex than needed, less appropriate for the operational environment, and more prone to failure.

This paper presents point-of-departure Sustainable System Mass evaluation criteria for ECLSS technologies that may, once refined, allow for integration of sustainability criteria into ESM evaluations and even allow ESM to be used to compare technologies at different TRLs. This paper encourages readers to contribute to evolving these concepts and criteria.

An example ECLSS water recovery technology is also presented that may embody some of the sustainability criteria presented. This example and others should be further developed with collaborators to enable more robust, sustainable technologies. These technologies may draw on terrestrial applications; and likewise, sustainable ECLSS technologies may be applied to life support challenges on Earth.

\section{Acknowledgments}

The author thanks John Graf, Ph.D. for coaxing the development of this concept.

\section{References}

${ }^{1}$ NASA's Exploration Systems Architecture Study, TM-2005-214062, NASA, 2005.

${ }^{2}$ Levri, J., Vaccari, D., and Drysdale, A. "Theory and Application of the Equivalent System Mass Metric," International Conference Environmental Systems2000, SAE: Toulouse, France.

${ }^{3}$ Drysdale, A., Ewert, M., and Hanford, A. "Life Support Approaches for Mars Missions." Advances in Space Research, Vol. 31, No. 1, 2003, pp. 51-61.

${ }^{4}$ Thomas, E., Leidich, J., and Klaus, D. "A Sustainable Regolith-Based Water Recovery Concept for the Lunar Outpost," ICES2009, SAE: Savannah, Ga. 


\section{Acronyms}

CFF: $\quad$ critical failure frequency

ECLSS: $\quad$ Environmental Control and Life Support System

ESM:

equivalent system mass

ISRU: in-situ resource utilization

KPP: $\quad$ key performance parameter

LEED: $\quad$ Leadership in Energy and Environmental Design

ORU: $\quad$ on-orbit replaceable unit

SECLSS: $\quad$ Sustainable Environmental Control and Life Support System

SSM: $\quad$ sustainable system mass

TRL: $\quad$ Technology Readiness Level

WRS: Water Recovery System 